\title{
Performance of Multichannel CSMA Networks
}

\author{
F. L. Lo \\ fllo@hkueee.hku.hk \\ T. S. Ng \\ tsng@hkueee.hku.hk \\ T. I. Yuk \\ tiyuk@hkueee.hku.hk \\ Department of Electrical and Electronic Engineering \\ The University of Hong Kong, HONG KONG
}

\begin{abstract}
Channel sense multiple access with collision detection (CSMA/CD) is a very simple and efficient way of allowing many stations to transmit messages to a central server down a shared channel. In wireless networks, however, collision detection is difficult to implement, and in such cases CSMA alone may have to be used. In this paper, it is shown that a multichannel CSMA network can be almost as efficient in utilizing the bandwidth available to the network as an equivalent single-channel CSMA/CD network. Furthermore, multichannel CSMA networks provide better throughput and delay performance than equivalent single channel CSMA systems, even when the message generation probability and the number of stations in the network are varied.
\end{abstract}

\section{INTRODUCTION}

When many stations need to communicate with a central server, but at very infrequent and non-periodic intervals, a non-centralized multiple access protocol for the contention of the shared channel is usually used. The first of this form of access protocol, or queue discipline, ALOHA [1], is very simple to implement, but unfortunately gives a very low channel utilization efficiency of only about $18 \%$ [2]. A much more efficient way of allowing stations to contend for a shared channel is CSMA/CD [3], as it prevents stations from transmitting when the channel is in use, and also avoid wasting channel bandwidth when message collisions occur. With CSMA/CD, channel utilization can be pushed up to beyond $90 \%$ [4]. Collision detection, however, is difficult to implement in a wireless network, because signal strength drops off very rapidly with distance [5]. A station that is transmitting, if it monitors the channel, will not hear transmissions from any other stations because of its own overwhelmingly strong signal. For wireless networks, CSMA seems like the best possible multiple access protocol to use.

It was shown in [6] that multichannel fullyconnected networks will perform better than equivalent single channel systems. Therefore, one possible way of increasing the throughput of a star-connected CSMA network may be to divide up the available bandwidth in the system and create several channels, either by FDMA, TDMA or CDMA. In this paper the performance of a multichannel star-connected CSMA network is obtained using equilibrium point analysis (EPA). It will be shown that a multichannel CSMA network can provide almost as high a channel utilization efficiency as an equivalent single channel CSMA/CD network, provided that the retransmission probability is chosen carefully. Furthermore, it is also shown that a multiple channel CSMA network does indeed provide a better throughput-delay performance than an equivalent single channel CSMA system.

The model for the multichannel network is described in Section 2, and the analysis is provided in Section 3. Some numerical results are presented in Section 4 , and the paper ends with some conclusions in Section 5.

\section{NETWORK MODEL}

The system has $N$ stations, which can use any of $M$ channels to transmit messages to a central server. The channels all have equal bandwidth, and time is divided into slots. Stations with no messages to transmit are called idle, and idle stations are presented with new messages at the rate of $s$ per slot. We shall take $s<1$, so $s$ can also be treated as the message generation probability for an idle station per time slot. When an idle station is presented with a message during a time slot, it senses all the $M$ channels. Should one or more channels be sensed free, i.e., with no on-going transmissions, the station will choose one of the free channels randomly with equal probability, and transmit down it during the next time slot. If no other station try to transmit down the same channel during that slot, the station is said to have captured the channel, as all the other stations will be able to sense the signal energy in the channel in subsequent slots and refrain from transmitting down it, and can continue transmitting until the end of the message. Messages are assumed to be made up of a random number of packets, each taking exactly one time slot to transmit, and this random number is 
geometrically distributed with mean $l$. Hence once a station has started transmitting, the probability that it will complete transmission during each subsequent slot is $1 / l$.

Should two or more stations attempt transmissions down the same channel during the same slot, a collision is said to occur, and the stations are assumed to be aware of it only at the completion of their unsuccessful message transmission, either when they do not receive an acknowledgment from the central server, or when they sense that the channel in the slot immediately after they have stopped transmission is not silent. All stations with an unsuccessfully transmitted message will be called blocked. Stations can become blocked either because they were involved in collisions, or because no channels were free when they were presented with new messages, and blocked stations will not accept newly generated messages until they become idle again. Blocked stations will sense all the channels during each slot subsequent to becoming blocked, and when one or more channels are sensed free, will attempt retransmission with probability $p$ down one of the free channels chosen randomly.

The system is assumed to be ideal, and all imperfections such as thermal noise, other forms of interference, timing jitters and multi-path fading are assumed to be insignificant. Messages that do not collide are therefore always received correctly, and signals in channels are always correctly sensed, so no stations will attempt transmissions down captured channels.

\section{ANALYSIS OF MODEL}

Although an exact Markov analysis can be performed on the system as described above, because of the large number of variables that need to be specified for each system state, the amount of computation that is needed to find the transition matrix, and later to solve for the stationary probabilities, will be formidable. Simulation of the network can also be attempted to obtain its throughput and delay, but unless it is known that the system is stable, as defined in [7], the figures obtained may just indicate the performance of the system in one of its several stable operating points.

We shall therefore use equilibrium point analysis to study the multichannel CSMA networks. Equilibrium point analysis (EPA), first used in [7], and called 'fluid-flow approximation' there, and explained in detail in [8], allows the approximate average behavior of a large system to be obtained with relatively little work, and also allows one to determine if the system is stable. The idea behind EPA is that the system will stay close to the point, or points, in system state space where the change in the different variables in the system are balanced. If there is only one stable point, the equilibrium throughput and delay can be easily obtained by finding this point, and these can then be treated as approximations of their averages. For systems having many stations with only one equilibrium point, the equilibrium values are very close to the average values, as shown in [8].

First we define some terms to help in our later discussions. The number of idle stations during a time slot will be denoted by $n_{0}$, of blocked stations by $n_{b}$, of colliding stations $n_{c}$, and of successfully transmitting stations by $n_{t}$. The number of free channels will be denoted by $m_{f}$, of captured channels by $m_{t}$, and of channels involved in collisions by $m_{c}$. Thus

and

$$
n_{0}=N-n_{t}-n_{b}-n_{c}
$$

$$
m_{f}=M-m_{t}-m_{c} .
$$

Also, since each successfully transmitting station has captured exactly one channel,

$$
n_{t}=m_{t} .
$$

The message input rate is

$$
S_{\text {in }}=n_{0} s
$$

and the message output rate

$$
S_{\text {out }}=\frac{n_{t}}{l} .
$$

When the system has message input and output rates equal, then

$$
n_{t}=\frac{\left(N-n_{b}-n_{c}\right) l s}{l s+1} .
$$

The average number of channels captured, given that there are $m_{f}$ free channels is

$$
\begin{aligned}
& S_{c a p}\left(m_{f}\right)=\sum_{c=1}^{m_{f}} \sum_{a=0}^{n_{0}} \sum_{b=0}^{n_{b}}\left(\begin{array}{c}
n_{0} \\
a
\end{array}\right) s^{a}(1-s)^{n_{0}-a} \cdot \\
&\left(\begin{array}{c}
n_{b} \\
b
\end{array}\right) p^{b}(1-p)^{n_{b}-b} c P\left(m_{f}, a+b: c\right)
\end{aligned}
$$

where $P(m, n: k)$ is the probability that $k$ channels will be captured if $n$ stations attempt transmission and there are $m$ free channels, and $\left(\begin{array}{l}n \\ r\end{array}\right)=\frac{n !}{(n-r) ! r !}$. The probability $P(m, n: k)$ can be calculated as shown in the Appendix.

Eqn. 7 works fine if $m_{f}, n_{0}$ and $n_{b}$ are all integers as $P(m, n: k)$ is obtained exactly only for integral values of $m, n$ and $k$. In EPA, all the values are 
treated as real, and therefore eqn. 7 cannot be used as it stands. We can try to interpolate values for $P(m, n: k)$, when $m, n$ and $k$ are not integers, but this is not straightforward. What we shall do instead is to assume that systems always stay very close to the equilibrium points. Should $m_{f}$ not be an integer, we take it that for $m_{f}-\left\lfloor m_{f}\right\rfloor$ fraction of the time, $\left\lceil m_{f}\right\rceil$ channels are free, and for the rest of the time, $\left\lfloor m_{f}\right\rfloor$ channels are free, where $\lfloor x\rfloor$ denotes the largest integer not greater than $x$ and $\lceil x\rceil$ denotes the smallest integer greater than $x$. Thus if $m_{f}=1.2$, then we assume that the system has 1 channel free $80 \%$ of the time, and 2 channels free $20 \%$ of the time.

With this assumption, the average capture rate can then be taken as

$$
\begin{gathered}
S_{c a p}=\left(m_{f}-\left\lfloor m_{f}\right\rfloor S_{c a p}\left(\left[m_{f}\right\rceil+\right.\right. \\
\left(\left[m_{f}\right\rceil-m_{f}\right) S_{c a p}\left(\left\lfloor m_{f}\right\rfloor\right.
\end{gathered}
$$

At equilibrium, the channel capture rate must be equal to the average message output rate, since each channel capture yields exactly one message successfully transmitted, so

$$
S_{c a p}\left(m_{f}\right)=\frac{n_{t}}{l} .
$$

The station collision rate given that there is at least one free channel is

$$
\begin{gathered}
C\left(m_{f}\right)= \\
\sum_{c=1}^{m_{f}} \sum_{a=0}^{n_{0}} \sum_{b=0}^{n_{b}}\left(\begin{array}{c}
n_{0} \\
a
\end{array}\right) s^{a}(1-s)^{n_{0}-a}\left(\begin{array}{c}
n_{b} \\
b
\end{array}\right) p^{b} \bullet \\
(1-p)^{n_{b}-b}(a+b-c) P\left(m_{f}, a+b: c\right)
\end{gathered}
$$

At equilibrium, this station collision rate must be equal to the rate at which colliding stations finish their unsuccessful transmission and enter the blocked mode, so

$$
C\left(m_{f}\right)=\frac{n_{c}}{l} \text {. }
$$

There are 7 variables which must be specified to determine the state the system is in, and we have six equations which must be satisfied when the system is in equilibrium, namely equations $1-3,6,9$ and 11 . We need another equation in order to find the equilibrium point, and to get it, we assume that on average, a channel experiencing collisions has exactly 2 stations transmitting through it. Thus

$$
n_{c}=2 m_{c}
$$

This assumption is good when $s<p<<1$, because the probability of collisions involving more than 2 stations drop by at least a factor of $p$ for each additional station.

By finding the equilibrium point, we can find the equilibrium throughput $S_{e q}$ and also the equilibrium number of blocked and colliding stations $n_{b c}(e q)$. We shall take these equilibrium values as the average values, and by Little's Formula, the average delay is then given by

$$
d=\frac{n_{b c}(e q)}{S_{e q}}
$$

4

\section{RESULTS OF ANALYSIS}

Fig. 1 shows the throughput and delay of a single channel CSMA/CD and triple channel CSMA network with $N=40$ and $s=0.002$. As can be seen from Fig. 1a, the single channel CSMA/CD network has a maximum throughput of about 0.055 message/slot, which works out to be a channel utilization efficiency of about $82.5 \%$, at $p \approx 0.07$. The triple channel CSMA network, even though suffering from a low throughput when the retransmission probability is increased from about 0.02 , also has a maximum throughput of about $0.055 \mathrm{message} / \mathrm{slot}$, but at $p=0.015$. Thus, if the retransmission probability is chosen carefully, a multichannel CSMA network can provide almost as high a throughput as a single channel CSMA/CD system. Some simulation points are also shown in Fig. 1 for the CSMA network, and they agree well with the values obtained through EPA. Note that all simulations are carried over 500,000 time slots, and that the throughputs and delays for the single channel CSMA/CD network is obtained through the EPA method described in [9].

In a mobile computing environment, the message generation probability and the number of stations within the network may not be constant. Figs. 2 and 3 show the behavior of CSMA networks as $s$ and $N$ are varied.

Fig. 2 shows the throughput and delay values for CSMA networks with 1, 3 and 5 channels, $N=40$, and $p=0.02$, when the message generation probability is varied. For all the values of $s$ shown on the graphs, the throughputs of the multichannel networks are higher than those of the single channel system, and the delays lower. Thus even if there should be a rise in $s$, the multichannel CSMA networks will still provide better performance than equivalent single channel ones.

Fig. 3 shows the throughputs and delays of CSMA networks with message generation probability of 0.002 , retransmission probability of 0.02 and various values of $N$. Again for the range of $N$ shown, the 
multichannel systems provide higher throughputs and lower delays than corresponding single channel ones.

Note that to be able to compare the performance of two systems with a different number of channels on an equitable basis, we assume that the bandwidth used by both are the same. If one of the system has $M_{1}$ channels and each message takes an average of $l_{1}$ slots to transmit, then the second system which has $M_{2}$ channels will require $l_{2}=\frac{M_{2}}{M_{1}} l_{1}$ slots on average to transmit. In the cases illustrated by in all the 3 figures, the single channel system has an average message length of 15 packets. Hence the three and five channel systems have average message lengths of 45 and 75 packets respectively.

\section{CONCLUSIONS}

This paper described a way to find the approximate throughput and delay of multichannel CSMA networks when there is a single equilibrium point. Using it, a multichannel CSMA network is shown to provide almost as good a performance as an equivalent single channel CSMA/CD network, provided that the retransmission probability is carefully chosen. It was also shown that multichannel CSMA networks provide higher throughputs and lower delays than equivalent single channel CSMA systems, even when the message generation probability and the number of stations in the system are varied.

One last point to note is that we assume the dividing up of the available system bandwidth into several channels incur no costs. In practical cases, the division will not be perfect, and for example, if FDMA is used, some bandwidth will have to be sacrificed as guard-bands. But if the number of channels is kept small, such bandwidth wastage can be kept to a minimum.

\section{APPENDIX}

If there are $m$ free channels and $n$ stations attempting to transmit during a slot, then the number of ways none of the stations that attempted transmission will capture a channel is

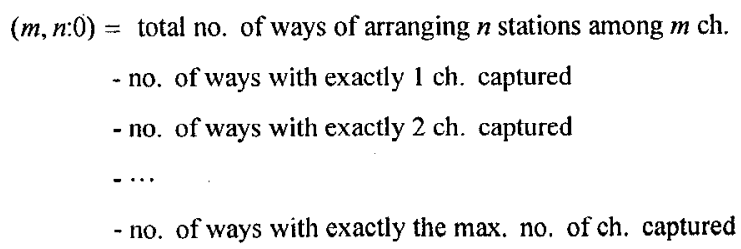

The maximum number of channels that can be captured is

$$
m_{\max }=\left\{\begin{array}{cc}
n & \text { if } n \leq m \\
m-1 & \text { if } n>m
\end{array}\right.
$$

The total number of ways of arranging $n$ stations among $m$ channels is $m^{n}$, and the number of ways that exactly $k$ channels get captured if $n$ stations attempt transmission when $m$ channels are free is

$$
(m, n: k)= \begin{cases}0 & k>m, \text { or } k>n, \text { or } k=n-1 \\
0 & n-k \neq 0 \text { and } m-k=0 \\
\left(\begin{array}{l}
m \\
k
\end{array}\right)\left(\begin{array}{l}
n \\
k
\end{array}\right) k ! \bullet & \text { otherwise } \\
(m-k, n-k: 0) & \end{cases}
$$

Thus

$$
\begin{aligned}
& (m, n: 0)=m^{n}-(m \cdot n \cdot(m-1, n-1: 0)) \\
& -\left[\left(\begin{array}{l}
m \\
2
\end{array}\right)\left(\begin{array}{l}
n \\
2
\end{array}\right) 2 !(m-2, n-2: 0)\right] \\
& -\cdots \\
& -\left[\left(\begin{array}{l}
m \\
k
\end{array}\right)\left(\begin{array}{l}
n \\
k
\end{array}\right) k !(m-k, n-k: 0)\right] \\
& -\left\{\begin{array}{cc}
\left(\begin{array}{c}
m \\
n
\end{array}\right) n ! & \text { if } n \leq m \\
m\left(\begin{array}{c}
n \\
m-1
\end{array}\right)(m-1) & \text { if } n>m
\end{array}\right.
\end{aligned}
$$

All the $(m-k, n-k: 0)$ terms can be derived recursively from eqn. A.4, and with these any $(m, n: k)$ can be calculated from eqn. A.3.

Thus the probability that $k$ stations will capture $k$ of the $m$ free channels if $n$ stations attempt transmission is just

$$
P(m, n: k)=\frac{(m, n: k)}{m^{n}}
$$

\section{ACKNOWLEDGMENT}

This work was supported by the Hong Kong Research Grant Council and by the CRCG of The University of Hong Kong, Hong Kong.

\section{REFERENCES}


Communications", Proc. AFIPS Conf., 1970

Fall Joint Comput. Conf., Vol. 37, pp. 281-285, 1970.

[2] N. Abramson, "The Throughput of Packet Broadcasting Channels," IEEE Trans. Comm. Vol. COM-25, pp. 117-28, 1977

[3] F. Tobagi, V. Hunt, "Performance Analysis of Channel Sense Multiple Access with Collision Detection", Comp. Networks, Vol. 4, pp. 245259,1980

[4] D. Heyman, "An Analysis of the Carrier-Sense Multiple Access Protocol", The Bell System Technical Journal, Vol. 61, pp. 2023-2051, 1982

[5] K. Pahlavan, A. Levesque, Wireless Information Networks, John Wiley, New York, pp. 486-490, 1995

[6] M. Marsan, D. Roffinella, "Multichannel Local Area Network Protocols", IEEE JSAC, Vol. SAC-1, pp. 885-897, 1983

[7] L. Kleinrock, S. Lam, "Packet Switching in a Multi-access Broadcast Channel: Performance Evaluation", IEEE Trans. on Commun., Vol. COM-23, pp. 410-423, 1975

[8] S. Tasaka, Performance Analysis of Multiple Access Protocols, MIT Press, Boston, 1986

[9] F.L. Lo, T.S. Ng, T.I. Yuk, "Performance Analysis of a Fully-Connected, Full-Duplex CDMA ALOHA Network with Channel Sensing and Collision Detection", IEEE JSAC, Vol. SAC-14, pp. 1708-1716, 1996

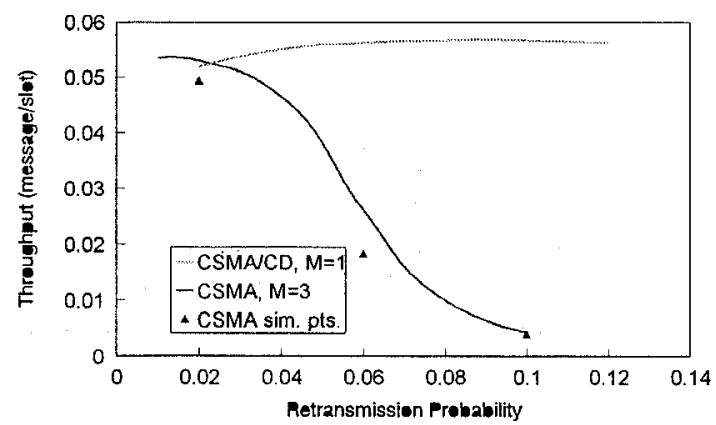

Fig.1a: Throughput against retransmission probability for systems with $N=40$ and $s=0.002$.

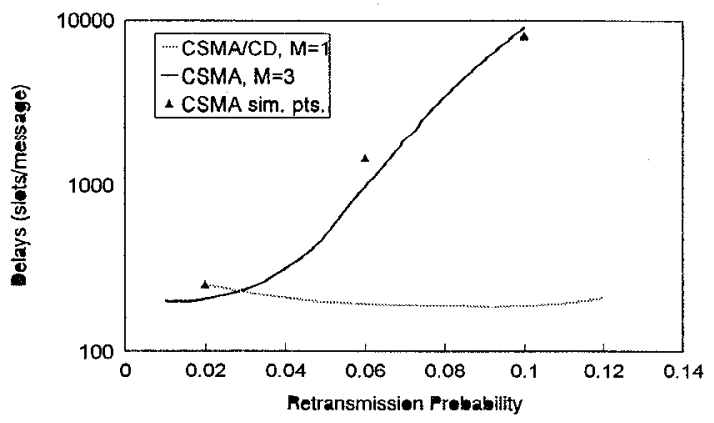

Fig. 1b: Graph of delays against retransmission probability

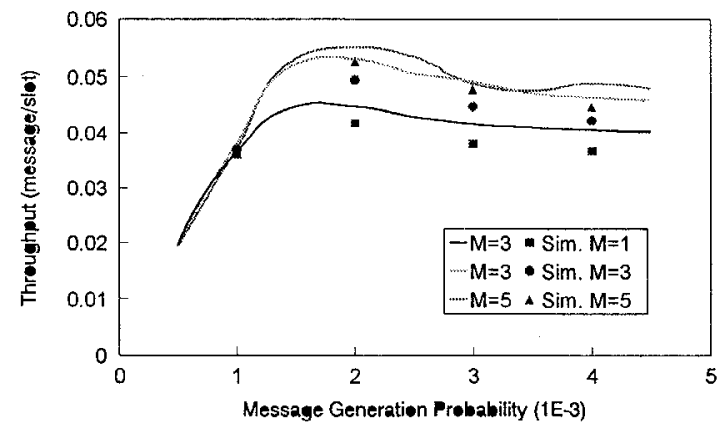

Fig. 2a: Graph of throughputs for CSMA systems with $p=0.02$, $N=40$.

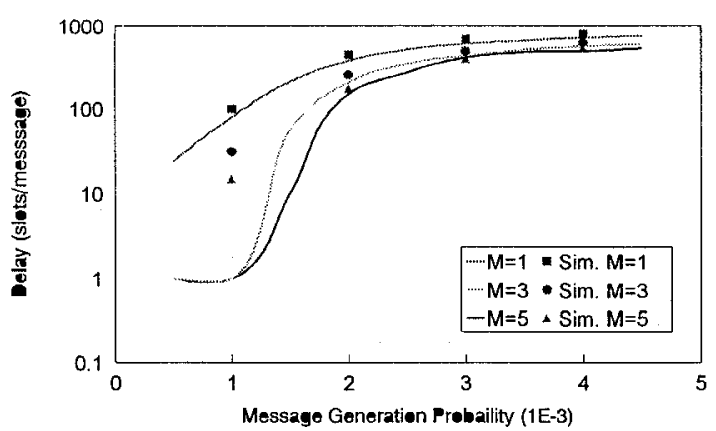

Fig. 2b: Graph of delays against message generation probabilty



Fig. 3a: Graph of throughputs for systems with $s=0.002, p=0.02$.

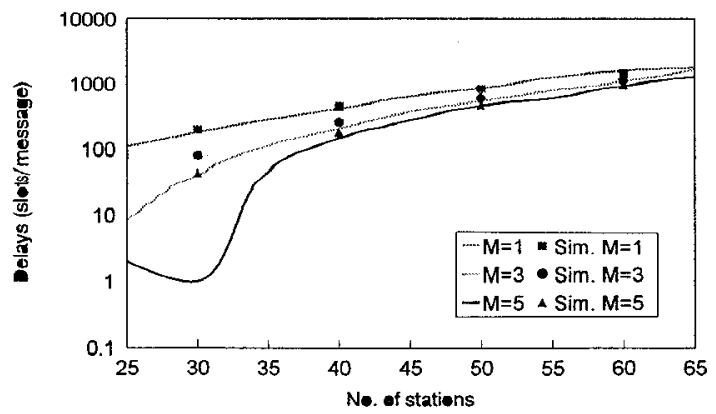

Fig. 3b: Graph of delays for systems with different number of stations. 